OPEN ACCESS

Edited by:

Xiaochao Xiong,

Washington State University,

United States

Reviewed by:

Xiaoming Tan,

Hubei University, China

Changhong Yao,

Sichuan University, China

Dennis Dienst,

Uppsala University, Sweden

${ }^{*}$ Correspondence:

Mary Ann Madsen

MaryAnn.Madsen@glasgow.ac.uk

Specialty section:

This article was submitted to

Bioprocess Engineering,

a section of the journal

Frontiers in Bioengineering and

Biotechnology

Received: 19 October 2020 Accepted: 09 December 2020

Published: 19 January 2021

Citation:

Madsen MA, Hamilton $G$ Herzyk P and Amtmann A (2021)

Environmental Regulation of PndbA600, an Auto-Inducible Promoter for Two-Stage Industrial Biotechnology in Cyanobacteria. Front. Bioeng. Biotechnol. 8:619055. doi: 10.3389/fbioe.2020.619055

\section{Environmental Regulation of PndbA600, an Auto-Inducible Promoter for Two-Stage Industrial Biotechnology in Cyanobacteria}

\author{
Mary Ann Madsen ${ }^{*}$, Graham Hamilton ${ }^{2}$, Pawel Herzyk ${ }^{1,2}$ and Anna Amtmann1 \\ ${ }^{1}$ College of Medical, Veterinary and Life Sciences, Institute of Molecular, Cell and Systems Biology, University of Glasgow, \\ Glasgow, United Kingdom, ${ }^{2}$ Glasgow Polyomics, Wolfson Wohl Cancer Research Centre, University of Glasgow, Glasgow, \\ United Kingdom
}

Cyanobacteria are photosynthetic prokaryotes being developed as sustainable platforms that use renewable resources (light, water, and air) for diverse applications in energy, food, environment, and medicine. Despite the attractive promise that cyanobacteria offer to industrial biotechnology, slow growth rates pose a major challenge in processes which typically require large amounts of biomass and are often toxic to the cells. Two-stage cultivation strategies are an attractive solution to prevent any undesired growth inhibition by de-coupling biomass accumulation (stage I) and the industrial process (stage II). In cyanobacteria, two-stage strategies involve costly transfer methods between stages I and II, and little work has been focussed on using the distinct growth and stationary phases of batch cultures to autoregulate stage transition. In the present study, we identified and characterised a growth phase-specific promoter, which can serve as an auto-inducible switch to regulate two-stage bioprocesses in cyanobacteria. First, growth phase-specific genes were identified from a new RNAseq dataset comparing two growth phases and six nutrient conditions in Synechocystis sp. PCC 6803, including two new transcriptomes for low Mg and low K. A type II NADH dehydrogenase $(n d b A)$ showed robust induction when the cultures transitioned from exponential to stationary phase growth. Behaviour of a 600-bp promoter sequence (PndbA600) was then characterised in detail following the expression of PndbA600:GFP in Synechococcus sp. PCC 7002. Culture density and growth media analyses showed that PndbA600 activation was not dependent on increases in culture density per se but on $\mathrm{N}$ availability and on another activating factor present in the spent media of stationary phase cultures (Factor $X$ ). PndbA600 deactivation was dependent on the changes in culture density and in either $\mathrm{N}$ availability or Factor $\mathrm{X}$. Electron transport inhibition studies revealed a photosynthesis-specific enhancement of active PndbA600 levels. Our findings are summarised in a model describing the environmental regulation of PndbA600, which can now inform the rational design of two-stage industrial processes in cyanobacteria.

Keywords: cyanobacteria, biotechnology, two-stage cultivation strategy, stationary phase, promoter, transcriptomics, nutrient limitation 


\section{INTRODUCTION}

Cyanobacteria are being developed as sustainable platforms that use renewable resources (light, water, and air) for diverse industrial applications, including the manufacturing of commodity and high-value products and remediation of heavy metals or salt (Amezaga et al., 2014; Al-Haj et al., 2016; Singh et al., 2016, 2017; Miao et al., 2020). This phylum of oxygenic photosynthetic bacteria inhabits virtually every niche across the planet and, coupled with its metabolic plasticity, lends itself to a vast variety of industrial settings and processes (Thajuddin and Subramanian, 2005; Xiong et al., 2017). Cyanobacteria produce a diverse palette of natural products with applications in energy, food, environment, and medicine, and are easily engineered for recombinant production (Camsund and Lindblad, 2014; Dittmann et al., 2015; Singh et al., 2017).

Despite the attractive promise that cyanobacteria offer to industrial biotechnology, they present unique challenges which have hampered its adoption by an industry currently dominated by well-established heterotrophic systems, such as Escherichia coli and Saccharomyces cerevisiae. While similarly amenable to highthroughput screening and engineering, many cyanobacterial strains still pose key technical difficulties such as cultivation and transformation-issues that have long been optimised for their heterotrophic competitors. With the collection of data and progress in technology, these barriers are gradually coming down. For instance, energetic and economic costs of cultivation and product purification are alleviated by rapid developments in photobioreactor and downstream processing technologies (Pierobon et al., 2018). Substantial effort has also been directed towards the development of molecular tools to genetically engineer these photosynthetic prokaryotes in which both standard prokaryote and photosynthetic eukaryote toolboxes are ineffective (Berla et al., 2013; Camsund and Lindblad, 2014; Santos-Merino et al., 2019). Despite the improvements in cultivation systems and the identification of relatively fast-growing cyanobacterial strains, however, slow growth rates continue to pose a major challenge to cyanobacterial biotechnology (Gale et al., 2019).

Industrial applications typically require a large biomass to obtain sufficient productivity. It is, therefore, important to avoid any growth inhibition during biomass accumulation in order to generate the optimal biomass as quickly as possible. In addition, computational analyses indicate trade-offs between biomass production and product synthesis in cyanobacteria (Knoop and Steuer, 2015). Two-stage cultivation strategies are, therefore, an attractive approach to decouple the industrial process (stage II) from biomass accumulation (stage I). Growth inhibition is thus minimised by alleviating issues arising from product/process toxicity, and stage I growth rates/stage II productivity is maximised by preferentially allocating resources (carbon precursors, ATP energy, and $\mathrm{NAD}(\mathrm{P}) \mathrm{H}$ reducing power) to growth or productivity, respectively (Burg et al., 2016).

In cyanobacteria, two-stage cultivation strategies typically require extra steps between stages I and II that add monetary and energetic costs to the process. The most common approach involves physical transfer of cultures from stage I to stage II, promoting conditions using centrifugation, filtration, flocculation, or sedimentation (Monshupanee et al., 2016; Kushwaha et al., 2018; Testa et al., 2019; Aziz et al., 2020). Alternatively, stage II can be induced by the application of physical and/or chemical stimuli such as changing light conditions for pigment production or temperature and salt stress for polysaccharide production in Spirulina (Lee et al., 2012, 2016). Strategies which eliminate these extra steps between stages I and II can greatly improve the economic feasibility of these systems.

Batch grown systems are well-suited for two-stage approaches. Bacterial growth is characterised by three successive phases: lag phase, exponential growth phase, and stationary phase. Cyanobacterial batch cultures show a similar growth pattern with the exception of an extended growth phase comprised of a shorter early growth phase, from which exponential growth rates are commonly reported, and a longer late growth phase, often termed the linear growth phase, as the cultures transition to the stationary phase (Schuurmans et al., 2017). Inherent differences between the growth and stationary phases of batch systems can be used to regulate two-stage processes and initiate stage II once maximum culture density has been achieved in the late growth/early stationary phase neither with any manipulation of culture nor with any added cost to the process.

Promoters are regulatory elements in the DNA that function as biological switches. Ideally, promoters controlling two-stage processes should be inactive during stage I and become active at the onset of stage II (Supplementary Figure S1). Auto-inducible promoters, which respond to endogenous signals, have the distinct advantage of not requiring any additional supplements, thus simplifying and improving the sustainability of the process. In the case of two-stage processes, promoters that specifically respond to changes in growth phase, particularly the transition to stationary phase, are ideal candidates. Libraries of stationary phase promoters have been developed for E. coli (Miksch et al., 2005). While orthogonal promoters derived from other organisms are generally preferred in order to avoid interference of engineered systems by host machinery, well-established prokaryotic tools perform poorly in cyanobacteria (Huang et al., 2010). In cyanobacteria, several growth phase-responsive genes and promoters have been reported for some model strains (Ludwig and Bryant, 2011; Berla and Pakrasi, 2012; Kopf et al., 2014; Ruffing et al., 2016). However, we still lack a detailed understanding of activation/deactivation behaviours and of performance across strains.

In this study, we aimed to identify and characterise an auto-inducible promoter for two-stage batch cultivation strategies in cyanobacteria using the approach presented in Figure 1. Two different species of cyanobacteria were used to avoid potential issues with genetic instability or cross-talk between native expression machinery and engineered expression systems (Camsund and Lindblad, 2014; Gordon and Pfleger, 2018). First, RNA sequencing analyses comparing transcriptional profiles across growth phases and nutrient conditions led to the identification of robust growth phase-specific genes and thus candidate promoters in the freshwater cyanobacterium Synechocystis sp. PCC 6803. Next, the behaviour of a promoter, PndbA600, was characterised in response to changing culture 


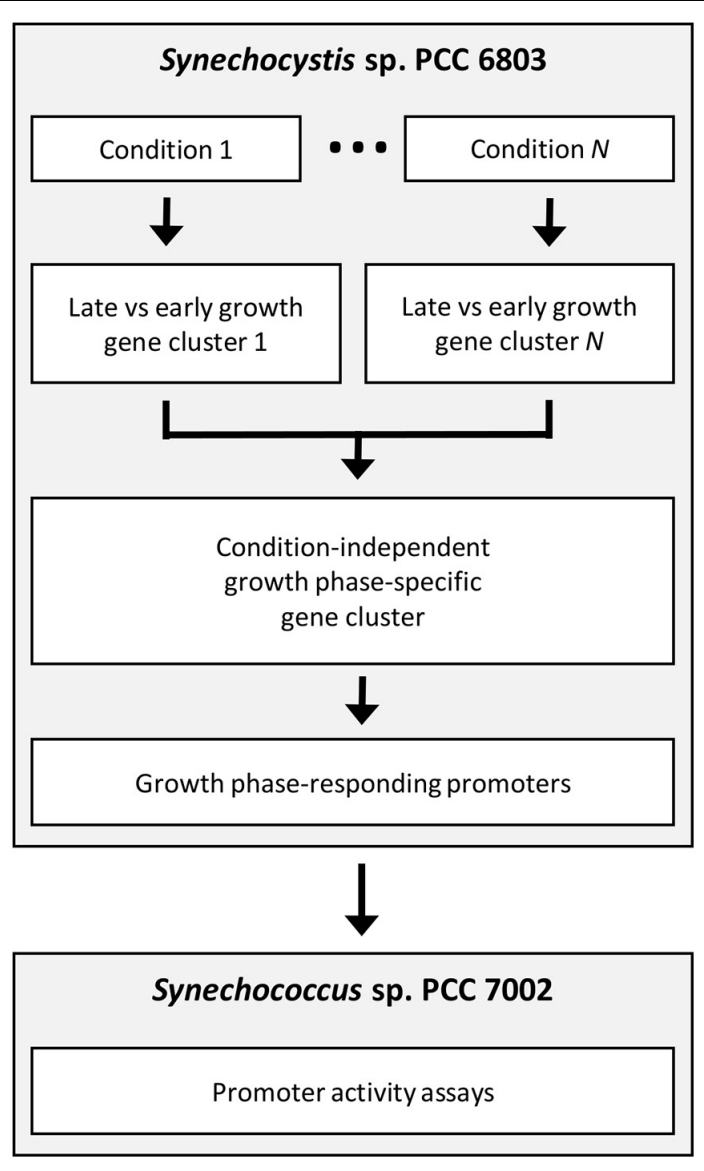

FIGURE 1 | Workflow for the identification and characterisation of growth phase-specific promoters. Synechocystis sp. PCC 6803 was cultivated in numerous different conditions. RNA sequencing of samples harvested during early and late growth phases led to the identification of condition-independent, growth phase-specific genes and candidate promoters. Heterologous promoter behaviour was characterised using a green fluorescent protein (GFP)-based assay in Synechococcus sp. PCC 7002.

density, growth media, and cellular redox status using a green fluorescent protein (GFP) assay in Synechococcus sp. PCC 7002. Our findings are summarised in a model describing the environmental regulation of PndbA600, which can inform the rational design of sustainable, two-stage industrial processes in cyanobacteria.

\section{MATERIALS AND METHODS}

\section{Strains and Culture Conditions Cyanobacterial Strains}

Synechocystis sp. PCC 6803 was grown photoautotrophically in Dreschel flasks in water baths equilibrated to $30^{\circ} \mathrm{C}$ with photoperiod $12 \mathrm{~h} / 12 \mathrm{~h}$ light/dark and light intensity $80 \mu \mathrm{mol}$ photons $\mathrm{m}^{-2} \mathrm{~s}^{-1}$. Synechococcus sp. PCC 7002 was grown photoautotrophically in Bijou bottles maintained at $30^{\circ} \mathrm{C}$ in a walk-in environmental growth chamber (Conviron model
MTPS72) with photoperiod $16 \mathrm{~h} / 8 \mathrm{~h}$ light/dark and light intensity $150 \mu \mathrm{mol}$ photons $\mathrm{m}^{-2} \mathrm{~s}^{-1}$. Cultures had a working volume of $60-75 \%$ relative to the culture vessel capacity, which were illuminated with fluorescent cool white lights and sparged with humidified ambient air.

\section{Growth Media}

For control conditions, Synechocystis cultures were grown in BG11 medium (Stanier et al., 1971) and Synechococcus cultures were grown in A + medium (Stevens and Porter, 1980). For low nutrient conditions, individual nutrients $(\mathrm{N}, \mathrm{P}, \mathrm{K}, \mathrm{S}$, and $\mathrm{Mg}$ ) were reduced to the indicated concentrations relative to the concentration in control BG11 or A + medium, and counter ions were replaced with control concentrations of $\mathrm{KCl}, \mathrm{MgCl}, \mathrm{Na}_{2} \mathrm{SO}_{4}$ or $\mathrm{NaH}_{2} \mathrm{PO}_{4}$ (see Supplementary Tables $\mathbf{S} 1$ and $\mathbf{S} 2$ respectively). To obtain "spent" media, the supernatant of stationary phase Synechococcus cultures (cultivated for $\geq 5$ weeks) was harvested after centrifugation at $4,000 \mathrm{~g}$ for $20 \mathrm{~min}$ at room temperature, and filter-sterilised.

\section{Culture Setup}

Twenty millilitre control medium was inoculated with strains maintained as DMSO stocks at $-80^{\circ} \mathrm{C}$ or on solid media maintained at $23^{\circ} \mathrm{C}$. For control and low nutrient conditions, $20 \mathrm{ml}$ cultures were grown to optical density (OD) $1-5$, diluted to $\mathrm{OD} 1$ in the relevant growth medium and $0.5 \mathrm{ml}$ was used to inoculate $150 \mathrm{ml}$ in the relevant growth medium. For promoter activation and deactivation experiments, 750 and $150 \mathrm{ml}$ cultures were grown in control conditions to low density $(\mathrm{OD}<5$, GFP $<300)$ and high density $(\mathrm{OD} \geq 12$, GFP $>850)$, respectively. Cells were harvested by centrifugation at $4,000 \mathrm{~g}$ for $20 \mathrm{~min}$ at room temperature, the supernatant was removed, and the pellets were washed and resuspended at OD 1 or 12 (for low and high density, respectively) in the relevant growth medium.

\section{Electron Transport Inhibitors}

3-(3,4-dichlorophenyl)-1,1-dimethylurea (DCMU, aka Diuron; Sigma) or malonic acid (Sigma) were added at the indicated concentrations to either young (1-week old cultures, OD $<5$, GFP $<300$ ) or mature (4-week old cultures, OD $\geq 12$, GFP > 850) $150 \mathrm{ml}$ cultures grown in control conditions.

\section{Growth Monitoring}

Growth was monitored by measuring $\mathrm{OD}$ at $730 \mathrm{~nm}\left(\mathrm{OD}_{730}\right)$ within the linear range (OD 0.05-1.00) of a Lambda 45 UV/VIS Spectrophotometer (PerkinElmer).

\section{RNA Analyses}

Total RNA was extracted using the RNeasy Mini Kit (Qiagen, Venlo, Netherlands). Frozen cell pellets were resuspended in $700 \mu \mathrm{l}$ Buffer RLT and cells were disrupted using $0.5 \mathrm{~g}$ of $0.5 \mathrm{~mm}$ diameter glass beads for $5 \mathrm{~min}$ at $30 \mathrm{~Hz}$ in a TissueLyser (Qiagen, Venlo, Netherlands). Following centrifugation at 10,000 $\mathrm{g}$ for 1 min, the supernatant was applied to the RNeasy spin column and RNA purified as recommended by the supplier.

For RNA sequencing, messenger RNA (mRNA) was enriched using the MICROBExpress Kit (Ambion, Austin, 
TX, United States). RNA quality was assessed before and after mRNA enrichment using an Agilent ${ }^{\circledR} 2100$ Bioanalyzer $^{\mathrm{TM}}$. Complementary DNA (cDNA) libraries were generated using TruSeq Stranded mRNA Library Prep Kit (Illumina) and sequenced using the Illumina MiSeq System at Glasgow Polyomics. Reads were processed and mapped to the Synechocystis genome (GenBank assembly accession GCA_000009725.1) using TopHat and Cuffdiff software (Trapnell et al., 2012). A total of 640,131,273 reads were obtained with average reads of 16.4 million reads per sample and an average length of 75 base pairs. A total of 546,270,878 reads $(85.3 \%)$ were mapped to the genome with a tolerance of a 2 base pair mismatch. The number of reads mapped to each coding sequence was calculated and normalised for gene length (number of fragments mapped per kilobase of gene) and library depth (total number of aligned reads in the experiment). Data are thus presented as fragments per kilobase of gene per million reads mapped (FPKM).

For each condition and time point, replicate samples from three independently grown cultures were sequenced, resulting in a total of 39 RNAseq datasets ( 1 control condition $\times 3$ time points $\times 3$ replicates +5 low nutrient conditions $\times 2$ time points $\times 3$ replicates). The raw RNA sequencing data are available from the European Nucleotide Archive, accession number PRJEB40560. Significant differences between conditions and time points were determined using Cuffdiff software (Trapnell et al., 2012). Multi-dimensional scaling of the RNA sequencing data was performed using the CummeRbund visualisation package (Trapnell et al., 2012).

For quantitative real-time PCR (qPCR), total RNA was isolated from different cultures to those used for RNA sequencing experiments, resulting in a total of 36 qPCR samples (6 nutrient conditions $\times 2$ time points $\times 3$ replicates). cDNA libraries were generated using random primers with the QuantiTect Reverse Transcription Kit (Qiagen, Venlo, Netherlands). qPCR was performed in a StepOnePlus Real-Time PCR System (Life Technologies). Primers were designed to amplify 110-155 bp products with a $60^{\circ} \mathrm{C}$ annealing temperature (Table 1) using Primer 3 software (Untergasser et al., 2012) and interrogated using Brilliant III Ultra-Fast SYBR Green QPCR Master Mix (Agilent). Primer pair efficiencies were determined using serial dilutions of gel purified RT-PCR product. Standard curves were

\begin{tabular}{|c|c|c|}
\hline $\begin{array}{l}\text { Target } \\
\text { gene }\end{array}$ & $\begin{array}{l}\text { Forward primer sequence } \\
\left(5^{\prime}-3^{\prime}\right)\end{array}$ & $\begin{array}{l}\text { Reverse primer sequence } \\
\left(5^{\prime}-3^{\prime}\right)\end{array}$ \\
\hline slr0211 & СCTGCTCCGGGCCTTGG & CTGGTATTGAATGGGGCCAC \\
\hline slr0451 & GAACAACAGGCCAGGGTAG & CGTAGTTCTTGCCGTTGGTG \\
\hline sll0401 & GAGAGTAGAAGCCGTTACCC & GCTGACGGAGAAGGAGCC \\
\hline slr1697 & CCCGATTTAACACCAATGTCC & GACTCAATATTGCTGGTAGCC \\
\hline slr0073 & GGAATATTGCACTCGTCTGGG & GCCAAGGTACGGTAGGAATG \\
\hline slr2144 & CAACAGTGACGGTCTGACC & CACCACTGCTTGCCCATCC \\
\hline psbA1 & СCTGTGGTCACGGTTCTGTT & TGCCATCAATATCCACCGGG \\
\hline$r n p B$ & GTGAGGACAGTGCCACAGAA & GATACTGCTGGTGCGCTCTT \\
\hline$n d b A$ & GACAAAAACGGTGCTCTGGG & CTCAAATCCGGGTTGACCAC \\
\hline slr1747 & GTTGCCCTCCСCTTGGTG & GAATATGGCTCGAATCCAACAC \\
\hline
\end{tabular}

included in all qPCR runs to transform threshold cycles into RNA concentrations, which were ratioed against the internal control slr0211. For replication, assays were performed using cDNA from three independently grown cultures. Significant differences between conditions and time points were determined by two-way ANOVA with Tukey (HSD) post-hoc analysis using SigmaPlot software (Systat). Negative (no template) controls were included and a melting curve analysis was performed in all assays.

\section{Promoter Analyses}

A $600 \mathrm{bp}$ sequence occurring directly upstream of the $n d b A$ gene, PndbA600, was amplified from Synechocystis genomic DNA using primers containing BioBrick prefix and suffix overhangs for cloning into the $\mathrm{pAQ} 1 \mathrm{BB}$ transformation vector: forward primer $5^{\prime}$ - gaattcgcggccgcttctagagTTAATGGATCGTTACCATTCCCAC $-3^{\prime}$ and reverse primer $5^{\prime}$ - ctgcagcggccgctactagtaAGCAACGGCG AAAATATTACGATTTG-3'. Following sequence confirmation in the pGEM-T Easy vector, PndbA600 was cloned upstream of a synthetic RBS-reporter construct comprised of RBS3 and GFP BioBrick part BBa_E0040 in the pAQ1BB vector (Madsen et al., 2018) to generate pAQ1BB:PndbA600:RBS3:GFP (Supplementary Figure S2). The sequence of the promoterreporter construct is presented in Supplementary Figure S3. The promoter-GFP construct was integrated into a neutral site in the Synechococcus genome by natural transformation and verified by PCR amplification and sequencing to generate the transgenic Synechococcus PndbA600:GFP strain. GFP was measured at regular intervals during culture growth by adjusting culture density to $\mathrm{OD}_{730} \quad 0.25-0.30$ and measuring fluorescence using $480 \mathrm{~nm}$ excitation and $514 \mathrm{~nm}$ emission wavelengths using a LS 55 Luminescence Spectrophotometer (PerkinElmer, Waltham, MA, United States).

\section{RESULTS}

\section{Environmental Conditions to Control Growth Phase Transition in Synechocystis Batch Cultures}

Stationary phase can be induced by many different factors including nutrient limitation, toxic by-product accumulation, or a variety of stress factors such as $\mathrm{pH}$ or temperature (Nyström, 2004). For robust separation of general responses to changes in the growth phase from specific responses to individual factors, several different conditions inducing transition to stationary phase were required. Nutrient deficiencies limit the growth of cyanobacteria (Hirani et al., 2001; Richaud et al., 2001) and were used here to induce the stationary phase. Synechocystis sp. PCC 6803 was cultivated under control conditions (BG11) and low levels of individual nutrients as reported before, with $12.5 \% \mathrm{~N}, \mathrm{P}$, or $\mathrm{S}$ in BG11 background. In addition, two new low nutrient conditions were tested, $12.5 \% \mathrm{Mg}$ and $12.5 \% \mathrm{~K}$ in BG11 background. Nutrients are co-supplied with a counter ion as an electrically neutral salt, so counter ions were replaced up to the control concentration (Supplementary Table S1). Figure 2A shows that Synechocystis sp. PCC 6803 batch cultures cultivated 

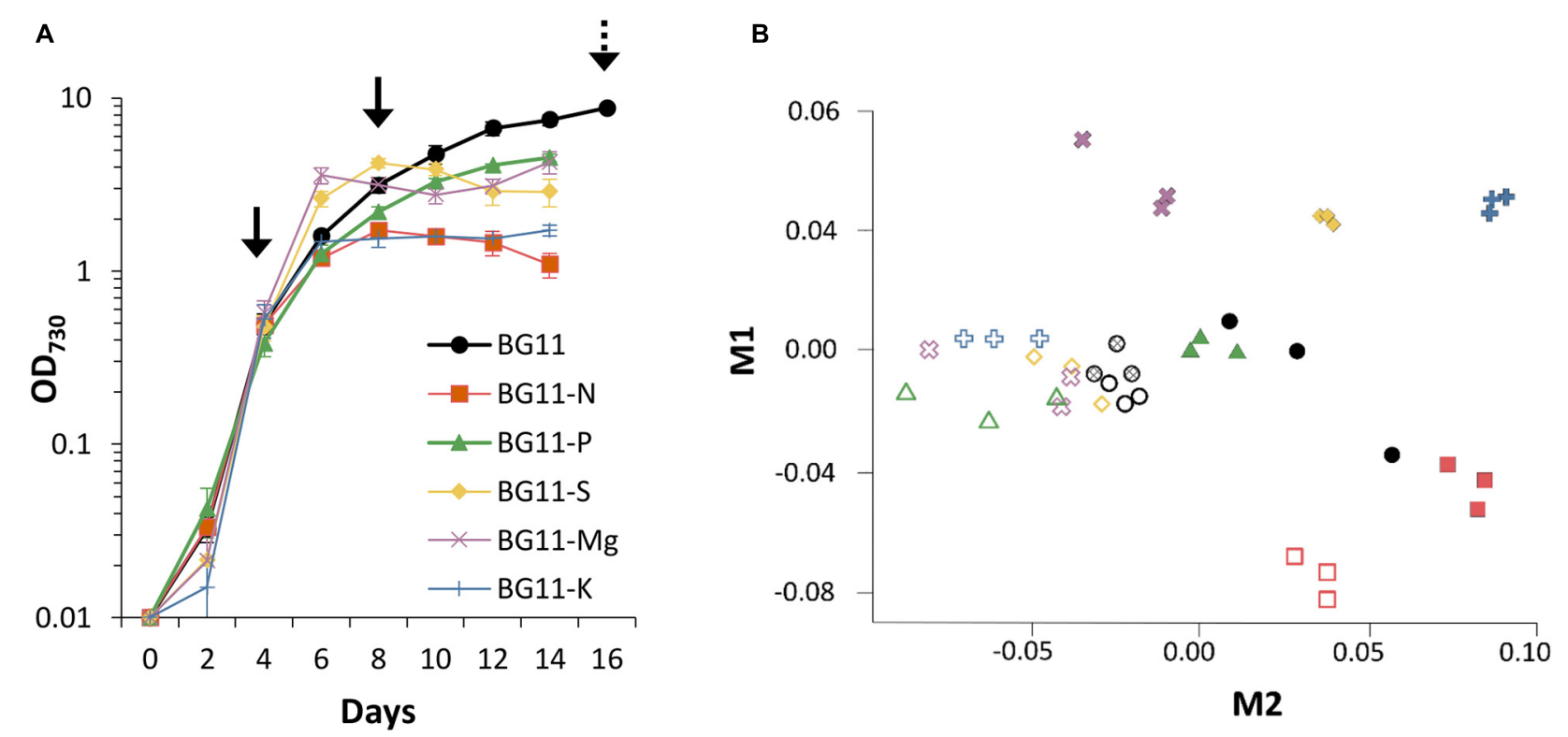

FIGURE 2 | Growth and transcriptomics of Synechocystis sp. PCC 6803. (A) Culture density (OD 730$)$ of Synechocystis batch cultures cultivated under control (BG11) and low nutrient conditions (12.5\% N, P, S, Mg, or K in BG11 background, Supplementary Table S1). Solid arrows show when samples were collected for RNA sequencing from all conditions. Dashed arrow indicates additional samples collected from control conditions. Data are means \pm S.E.M. of three independent cultures. (B) Multi-dimensional scaling plot of 39 RNAseq expression profiles. Distances between points reflect relative similarities between samples based on genome-wide transcript levels. Symbols and colours are same as in (A). Open symbols represent early growth phase samples (day 4). Closed symbols represent late growth phase samples (day 8 in low nutrient and day 16 in control conditions). Hatched circles represent control samples harvested on day 8.

in all five low nutrient conditions transition to the stationary phase at an earlier time (day 8$)$ and lower density $\left(\mathrm{OD}_{730} 1.5-4.2\right)$ compared to the control condition (day 16, $\mathrm{OD}_{730}$ 8.7). Thus, low nutrient conditions were used to induce early transition to the stationary phase in response to specific environmental stimuli.

\section{RNA Sequencing of Early and Late Growth Phases in Synechocystis}

To compare the transcriptomes of early and late growth phases, time points were selected for RNA sequencing based on the growth curves (black arrows in Figure 2A). Early samples were harvested during the exponential growth phase (day 4), and late samples were harvested as the cultures transitioned into the stationary phase (day 8). To account for later transition to the stationary phase, control cultures were also harvested on day 16. For replication, three cultures were independently grown in each condition. RNA purification and sequencing is described in Materials and Methods (Section "RNA Analyses"), and the normalised transcript levels for all genes in all 39 samples, together with statistical parameters, are available in Supplementary Table S3. Transcript counts are presented as FPKM.

Figure 2B shows a multi-dimensional scaling plot based on normalised FPKM values in the 39 RNAseq samples. Early samples generally group together with the exception of BG11$\mathrm{N}$, suggesting a distinct early response to low N. Additionally, samples harvested on day 8 under control conditions cluster with early samples, reflecting that nutrients were not yet limiting in this condition and control cultures were still in the early growth phase. Late samples separate according to condition with close grouping of replicates, demonstrating nutrient-specific transcriptional responses. Greater variation is observed in late control samples, possibly due to simultaneous limitation of multiple nutrients in the optimised BG11 medium in which nutrients deplete at equal rates.

\section{Robust, Late Growth Phase-Specific Genes of Synechocystis}

To identify condition-independent, growth phase-responsive genes, we looked for genes that were upregulated in the late growth phase compared to the early growth phase in all of the conditions tested. Genes were, therefore, selected from the RNA sequencing dataset based on a significance value of $p<0.05$ between early and late samples within each condition and $\log _{2}$ (late/early) $>1$ for all six conditions. Table 2 presents 24 late growth phase-specific genes from the Synechocystis RNA sequencing dataset. The majority of genes are annotated as hypothetical proteins (16/24, 67\%); however, there are also genes with annotated functions in cell killing, energy metabolism, photosynthesis and respiration, regulatory functions, and transport.

RNAseq expression profiles were verified using real-time qPCR. Three new Synechocystis cultures were grown for each of the six nutrient conditions, and samples were harvested during the early and late growth phases (36 samples total). To control for potential biases introduced during mRNA enrichment for the 
TABLE 2 | Late growth phase-specific genes of Synechocystis sp. PCC 6803.

\begin{tabular}{|c|c|c|c|}
\hline Gene ID & Gene symbol & Gene product & Functional category \\
\hline s/r1747 & & Cell death suppressor protein LIs1 homologue & Cellular processes \\
\hline slr2132 & & Phosphotransacetylase & Energy metabolism \\
\hline sll0549 & & Hypothetical protein & Hypothetical \\
\hline sll0528 & & Hypothetical protein & Hypothetical \\
\hline slr1119 & & Hypothetical protein & Hypothetical \\
\hline$s / 1675$ & & Hypothetical protein & Hypothetical \\
\hline s/17355 & & Hypothetical protein & Hypothetical \\
\hline s/11274 & & Hypothetical protein & Hypothetical \\
\hline s/l1769 & & Hypothetical protein & Hypothetical \\
\hline$s / 11158$ & & Hypothetical protein & Hypothetical \\
\hline s/l1884 & & Hypothetical protein & Hypothetical \\
\hline slr1674 & & Hypothetical protein & Hypothetical \\
\hline slr0959 & & Hypothetical protein & Hypothetical \\
\hline slr0292 & & Hypothetical protein & Hypothetical \\
\hline s/l6052 & & Hypothetical protein & Hypothetical \\
\hline sl/6053 & & Hypothetical protein & Hypothetical \\
\hline s/l6054 & & Hypothetical protein & Hypothetical \\
\hline s/l6055 & & Hypothetical protein & Hypothetical \\
\hline slr1498 & hypD & Putative hydrogenase protein HypD & Other categories \\
\hline slr1675 & hypA1 & Putative hydrogenase protein HypA1 & Other categories \\
\hline slr0851 & $n d b A$ & Type 2 NADH dehydrogenase & Photosynthesis and respiration \\
\hline slr0741 & & Transcriptional regulator & Regulatory functions \\
\hline slr0096 & & Low affinity sulphate transporter & Transport and binding proteins \\
\hline slr0529 & ggtB & Glucosylglycerol transport system substrate-binding protein & Transport and binding proteins \\
\hline
\end{tabular}

RNA sequencing analyses, cDNA for qPCR analyses was generated from total RNA. slr0211 (encoding a hypothetical protein) was selected as a reference gene for qPCR normalisation based on low standard error of FPKM across the 39 RNAseq samples and low variation of $\mathrm{Ct}$ values across the $36 \mathrm{qPCR}$ samples (Figure 3A). While there were small differences in the level of upregulation within individual conditions, there was an overall excellent agreement between RNAseq and qPCR expression profiles for $n d b A$ (slr0851, encoding a type II NADH dehydrogenase, Figures 3B,C) and slr1747 (encoding a homologue of the cell death suppressor protein Lls1, Supplementary Figure S4). While these genes are adjacent to one another on the Synechocystis chromosome, they are independent transcriptional units (Kopf et al., 2014), and both showed significantly higher transcript levels in the late growth phase compared to the early growth phase in all six nutrient conditions. The level of $n d b A$ upregulation was relatively consistent across the conditions tested; therefore, this gene was selected for promoter analyses.

\section{Heterologous Activity of the ndbA Promoter, PndbA600, in Synechococcus}

Heterologous systems have several advantages for the characterisation of molecular tools for metabolic engineering. For example, the interference from native regulatory machinery is minimised, such as small RNAs important for adaptation to changing environments in cyanobacteria ( $\mathrm{Hu}$ and Wang, 2018). Furthermore, introduction of high copy numbers of an endogenous, or even homologous, promoter could outcompete the native promoter (or vice versa) and potentially cause genetic instability in cyanobacteria (Gordon and Pfleger, 2018). Although there is a potential for common regulatory machinery, such as transcription factors across cyanobacteria, Synechocystis promoters are routinely used to drive the heterologous expression in Synechococcus sp. (Huang et al., 2010; Wang et al., 2012). We therefore used an established GFP-based promoter assay in Synechococcus sp. PCC 7002 (Madsen et al., 2018), to assess whether the $n d b A$ promoter controls growth phase-specific transcription. For this, we analysed the $600 \mathrm{bp}$ sequence directly upstream of the $n d b A$ start codon, which, in addition to the core promoter, includes the $5^{\prime} \mathrm{UTR}$ and other potential genetic features. This sequence was designated PndbA600 and cloned upstream of a GFP reporter gene and integrated into a neutral site in the Synechococcus genome. Following confirmation by PCR and sequencing, the transgenic strain Synechococcus PndbA600:GFP was grown in A + (control) medium, and GFP fluorescence per cell (GFP normalised to $\mathrm{OD}_{730}$ of the GFP sample) was measured throughout culture growth. Figure 4A shows that under control conditions the $\mathrm{OD}_{730}$-normalised GFP fluorescence was low during the early stages of growth followed by a sharp increase in week 3 , concomitant with transition to the late growth phase. High levels were then maintained over several weeks of the stationary phase. Notably, PndbA600 showed two distinct levels of the promoter activity with low activity at low density (OD < 10.7, GFP < 450) and high activity at high density (OD > 10.75, GFP 636-3590; Figure 4B). The GFP signal reflects 


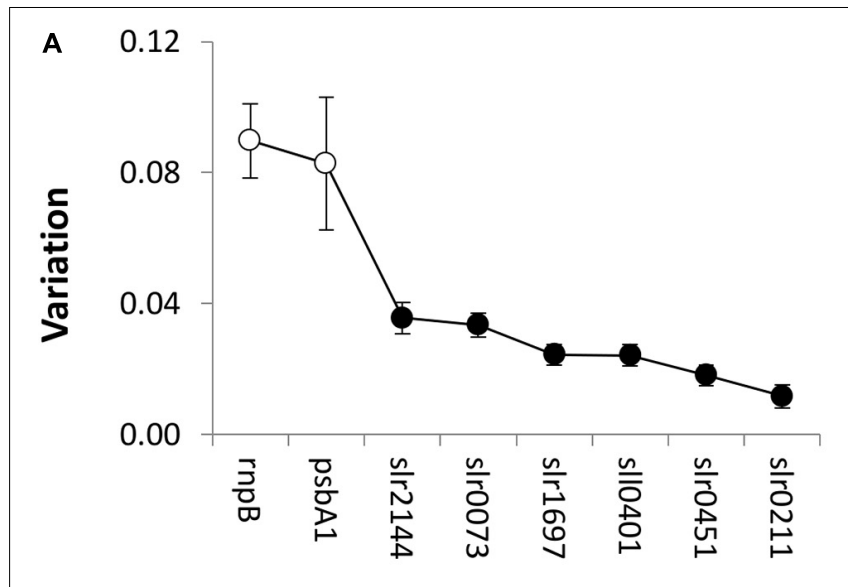

B

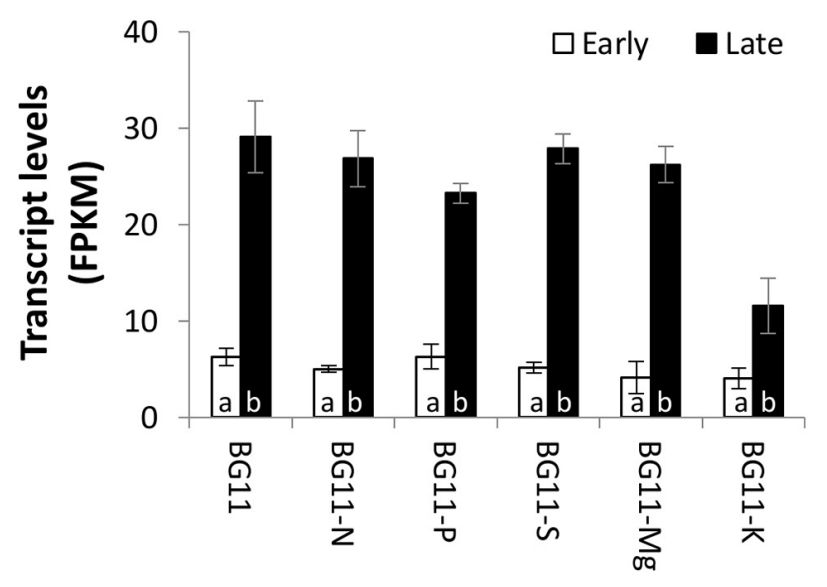

C

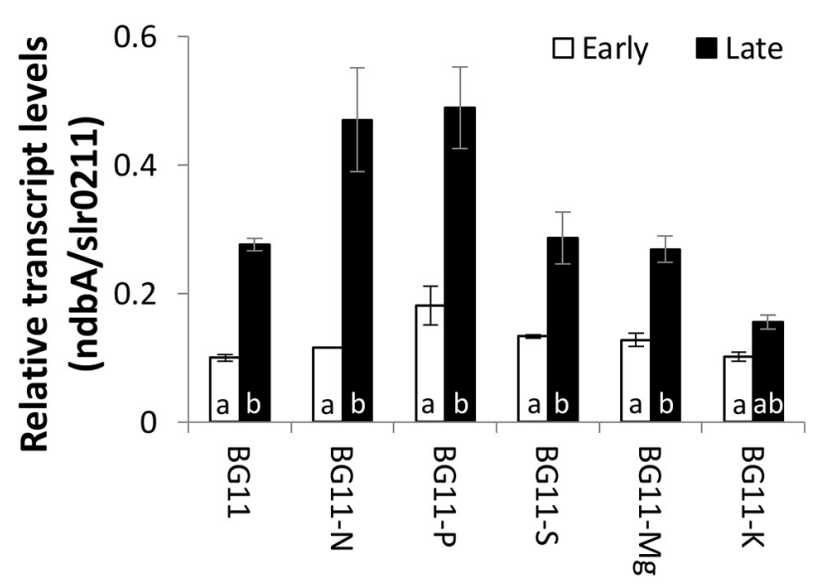

FIGURE 3 | Native ndbA transcript levels. (A) Variation of Ct values determined using NormFinder software (Andersen et al., 2004). qPCR was performed with 36 samples representing the early and late growth phases of three independent cultures of Synechocystis cultivated under control (BG11) and low nutrient conditions (12.5\% N, P, S, Mg, or K in BG11 background). White circles represent reference genes used in other studies (Foster et al., 2007; Pinto et al., 2012). Black circles represent genes selected from the RNA sequencing dataset based on low standard error. Data are means \pm S.E.M. of three independent cultures. slr0211 was selected as reference gene for further

(Continued)
FIGURE 3 | Continued

experiments. (B,C) Transcript levels of ndbA determined by (B) RNA

sequencing (normalised to gene length and read counts as FPKM) and (C) qPCR (normalised to slr0211) in the early (white bars) and late (black bars) growth phases of Synechocystis cultivated under control (BG11) and low nutrient conditions (12.5\% N, P, S, Mg, or K in BG11 background). Data are means \pm S.E.M. of three independent cultures. Different letters indicate significant difference across all conditions $(p<0.05$; two-way ANOVA using Tukey (HSD) post-hoc analysis).

PndbA600 promoter activity: background fluorescence levels in the wild-type and no promoter controls were lower than those observed in Synechococcus PndbA600:GFP (GFP < 120). These combined results show that the $600 \mathrm{bp}$ upstream sequence of $n d b A$, PndbA600, shows both growth phase- and culture density-specific activity in Synechococcus sp. PCC 7002 grown in control conditions.

\section{Effect of Culture Density on PndbA600 Activity}

Culture density, possibly due to direct cell-to-cell interactions, has been suggested as a factor controlling cell division and the transition into stationary phase in cyanobacteria (Esteves-Ferreira et al., 2017). Additional factors could include self-shading and thus light limitation in higher density cultures or extracellular metabolites e.g., signalling molecules secreted to the growth medium (Abisado et al., 2018; Clark et al., 2018). To investigate the relationship between culture density and promoter activity in more detail, we attempted to separate the effect of culture density from the effect of the growth medium. For this experiment, Synechococcus PndbA600:GFP cultures were grown to either low density to investigate PndbA600 activation or high density to investigate PndbA600 deactivation. Culture density was then modulated by harvesting the cells and resuspending them at either low or high density. Growth media were modulated by resuspending the cells in either a fresh (control) medium or a spent medium harvested from stationary phase cultures.

We first investigated PndbA600 activation using young, low density cultures with low promoter activity. Figure 5A shows that increasing culture density from low to high OD is not sufficient to activate PndbA600. GFP fluorescence only increased after a period of growth in the fresh medium for both low to low and low to high OD cultures (see also Supplementary Figure S5A). By contrast, resuspending low density cultures in the spent medium led to rapid PndbA600 activation with faster activation in cultures resuspended at low density compared to high density (Figure 5B and Supplementary Figure S5B). These results suggest that PndbA600 activation is not dependent on high culture density per se but requires one or more components of the spent stationary phase medium, designated as Factor X.

PndbA600 deactivation was then investigated using mature, high density cultures with high promoter activity. Figure 5C shows that decreasing culture density from high to low OD in 


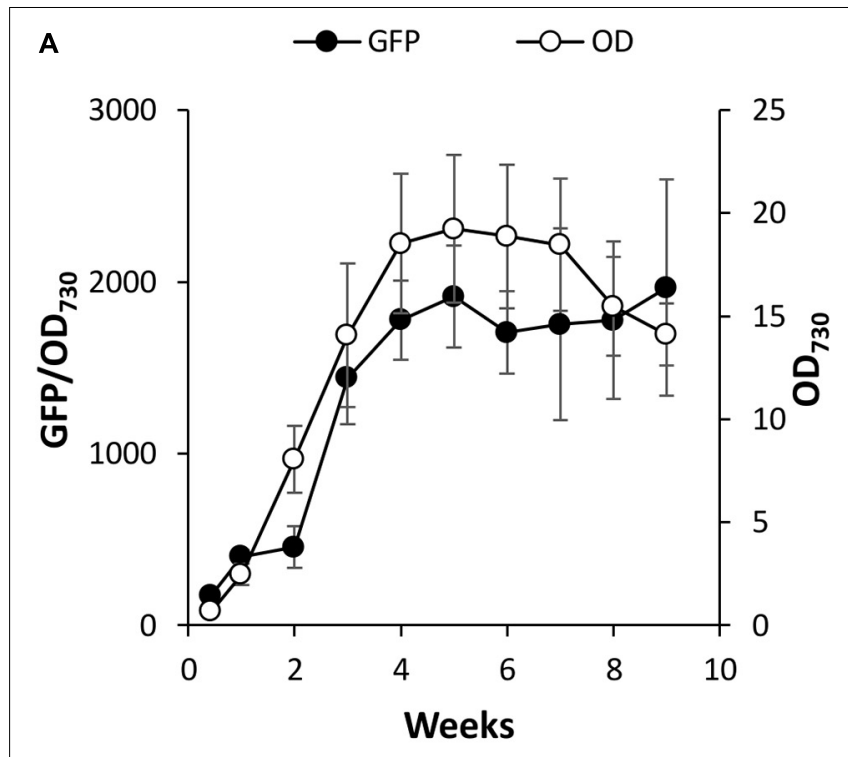

B $\square$ Wildtype $\triangle$ No promoter $\quad$ PndbA600

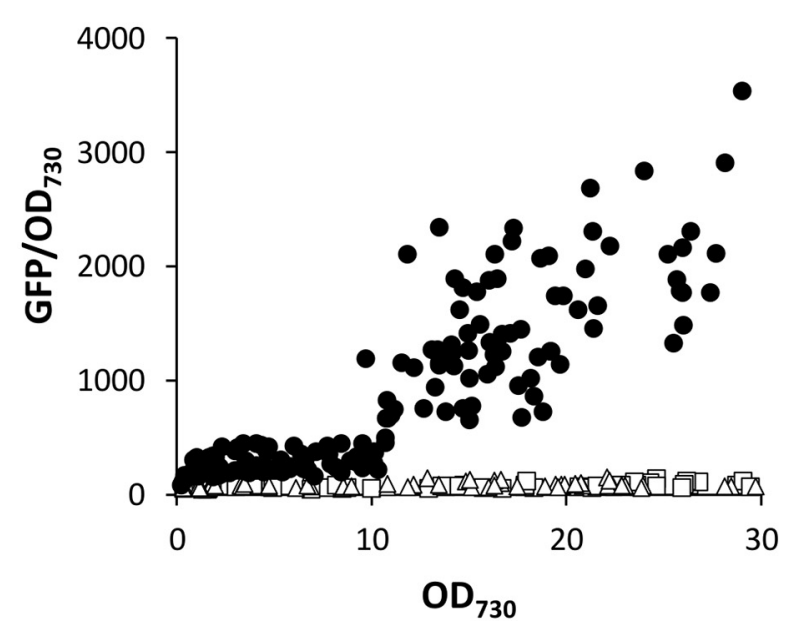

FIGURE 4 | Heterologous PndbA600 activity under control conditions. (A) Promoter activity (black circles, GFP fluorescence normalised to $\mathrm{OD}_{730}$, left axis) in Synechococcus PndbA600:GFP. Culture density ( $\mathrm{OD}_{730}$, right axis) is shown with white circles. Data are means \pm S.E.M. of three independent cultures grown in control A + media. (B) Promoter activity (GFP fluorescence normalised to $\left.\mathrm{OD}_{730}\right)$ against culture density $\left(\mathrm{OD}_{730}\right)$ of Synechococcus cultures grown in control conditions. Data are shown for the following lines: wild-type (no GFP, squares, $n=7$ ), no promoter (Synechococcus transformed with GFP, triangles, $n=6$ ), Synechococcus expressing GFP under the control of PndbA600 (Synechococcus PndbA600:GFP, circles). Measurements taken after maximum culture density was achieved are not shown.

a fresh medium resulted in a rapid loss of GFP fluorescence (within 1 week), indicating rapid deactivation of PndbA600. The promoter then regained activity as the culture moved again into the late growth phase (see also Supplementary Figure S5C). PndbA600 deactivation requires the decrease in cell density: mature, high density cultures resuspended at high density in fresh media maintained high promoter activity. PndbA600 deactivation also requires the fresh growth medium: mature, high density cultures resuspended at low density in spent media maintained high promoter activity (Figure 5D and Supplementary Figure S5D). This suggests that the alleviation of Factor $\mathrm{X}$ from the spent stationary phase medium is a co-requirement for PndbA600 deactivation. In summary, PndbA600 deactivation requires both low cell density and fresh growth medium.

\section{The Effect of Nutrient Availability on PndbA600 Activity}

We have shown that the spent medium of stationary phase cultures is able to modulate PndbA600 activity by both inducing promoter activation and inhibiting promoter deactivation. A possible explanation for these responses may be the low nutrient levels in the spent stationary phase medium. The native expression profile in Synechocystis suggested that PndbA600 activation in the late growth phase occurs independent of nutrient depletion. However, this might not be the case in Synechococcus. We, therefore, investigated the nutrient dependence of PndbA600 activity in Synechococcus PndbA600:GFP using A + with low levels of individual nutrients. Counter ions co-supplied with nutrients were replaced up to the control concentration (Supplementary Table S2). Unlike the optimised BG11 medium for Synechocystis, we found that nutrient ratios in the standard A + medium used for Synechococcus are not optimally adjusted, and different nutrients become limiting at different concentrations (Figure 6). Timing of PndbA600 activation and, thus, correlations between the promoter activity, culture density, and the growth phase differed across nutrient conditions.

When comparing the promoter activity relative to the growth phase transition, promoter activation occurred during the transition to the stationary phase at $>75 \%$ of maximum culture density under control conditions (Figure 4A). Lowering $\mathrm{N}$ in the growth medium accelerated PndbA600 activation, whereby promoter activation occurred during the active growth phase at $<50 \%$ of maximum culture density (Figure 6A). By contrast, PndbA600 activation was delayed in media with low $\mathrm{K}$ or low $\mathrm{Mg}$ with promoter activation first occurring $>1$ week after reaching maximum culture density (Figures 6B,C) and was completely abolished in low P (Figure 6D). In summary, PndbA600 activation, relative to the growth phase transition, is accelerated by lowering $\mathrm{N}$ and delayed by lowering other essential nutrients (and thus increasing the relative $\mathrm{N}$ level), suggesting that PndbA600 may be activated by the depletion of $\mathrm{N}$ relative to other nutrients in the media.

Plotting promoter activity directly against culture OD demonstrated promoter activation at high density $(\mathrm{OD}>10.75)$ under control conditions (Figure 4B). Figure 6E shows that PndbA600 activation occurred at lower culture densities in media with $20 \% \mathrm{~N}(\mathrm{OD}>1.84)$ and $2 \% \mathrm{~K}(\mathrm{OD}>6.15)$. By contrast, PndbA activation required a much higher culture density $(\mathrm{OD}>17)$ in media with $0.5 \% \mathrm{Mg}$. No PndbA activation was seen in $10 \% \mathrm{P}$ at any $\mathrm{OD}$. The varying culture OD 

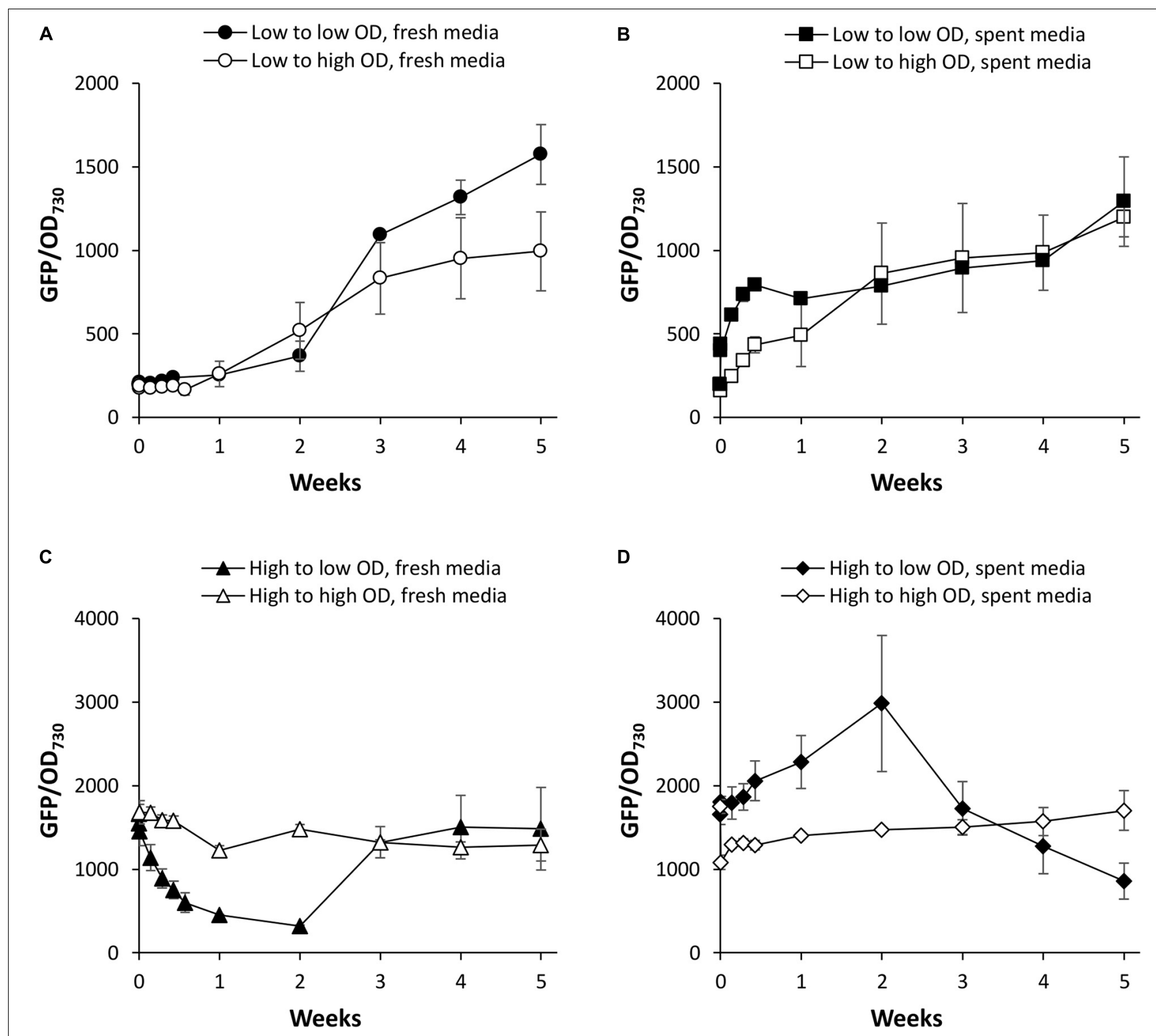

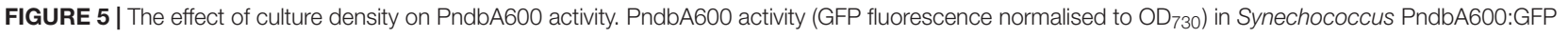
cultures pre-grown to certain density and resuspended to another density at time point 0 . Cultures shown in (A,B) were started from young, low density cultures with low promoter activity (OD < 5, GFP < 300). Cultures shown in (C,D) were started from mature, high density cultures with high promoter activity (OD > 12, GFP > 850). Cultures were resuspended to low (black symbols) or high (white symbols) density in fresh control media (A,C) or spent media of stationary phase cultures (B,D). Data are means \pm S.E.M. of three independent cultures. Accompanying growth curves are presented in Supplementary Figure S5.

requirements for PndbA activation under different low nutrient conditions reflect a combination of the maximum culture OD achieved and the timing of PndbA activation relative to the transition to stationary phase.

To investigate the effects of $\mathrm{N}$ supply in more detail, Synechococcus PndbA600:GFP cultures were grown in control conditions and resuspended at OD 1 in growth media containing either 0 or $100 \% \mathrm{~N}$ in a background of the control $\mathrm{A}+$ medium. Again, young Synechococcus PndbA600:GFP cultures grown to low density were used to investigate PndbA600 activation, and mature cultures grown to high density were used to investigate
PndbA600 deactivation. Figure 7A confirms that PndbA600 activation requires the lack of $\mathrm{N}$ : early promoter activation was observed in N-deficient media (see also Supplementary Figure S6A). Similarly, PndbA600 deactivation requires the presence of $\mathrm{N}$ : mature, high density cultures resuspended at low density in $\mathrm{N}$-deficient media maintained active levels of GFP fluorescence, albeit at a slightly lower level (Figure 7B and Supplementary Figure S6B). Combined, these results confirm that PndbA600 specifically responds to $\mathrm{N}$ levels, with promoter activation upon $\mathrm{N}$ depletion and promoter deactivation upon $\mathrm{N}$ replenishment. 


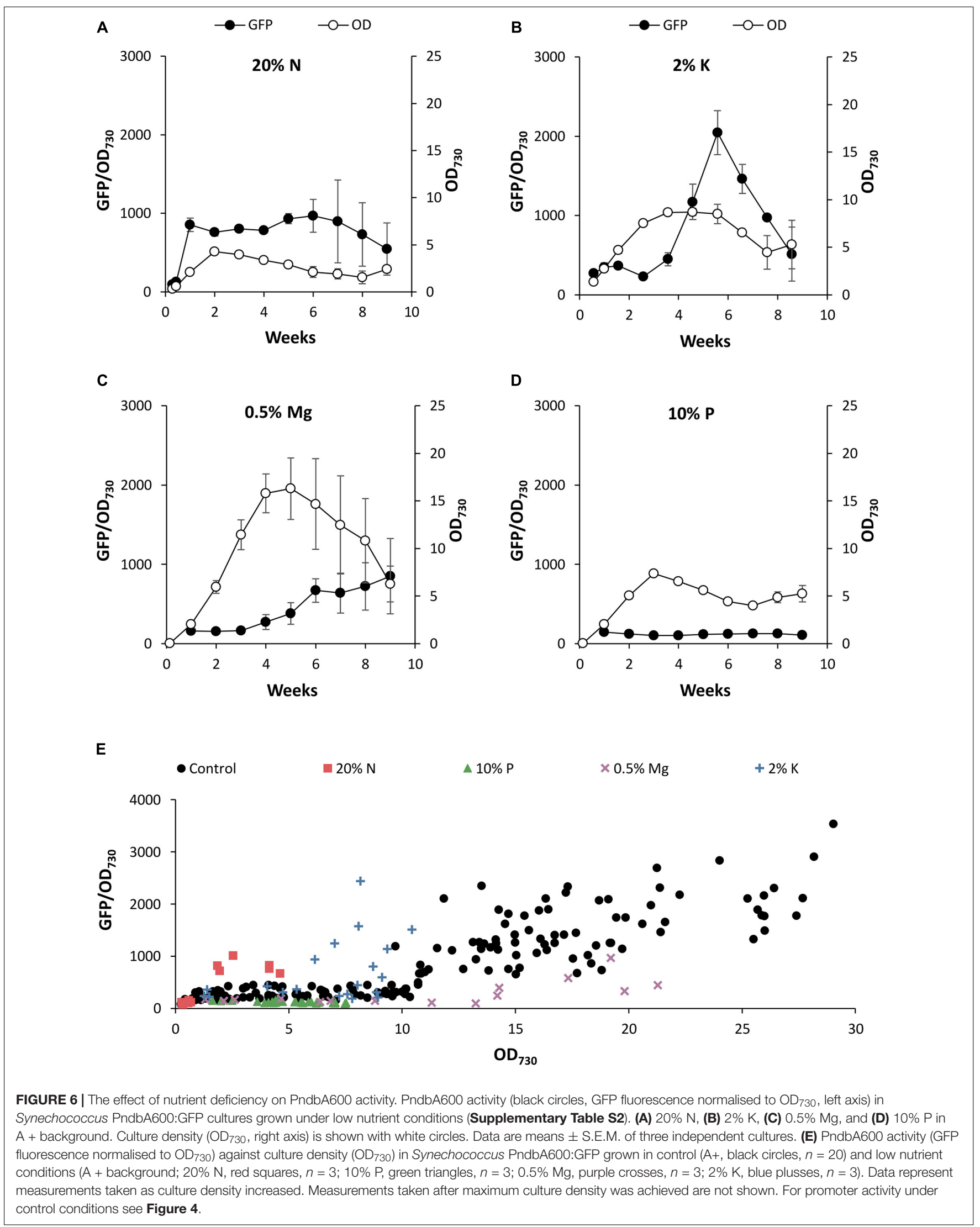



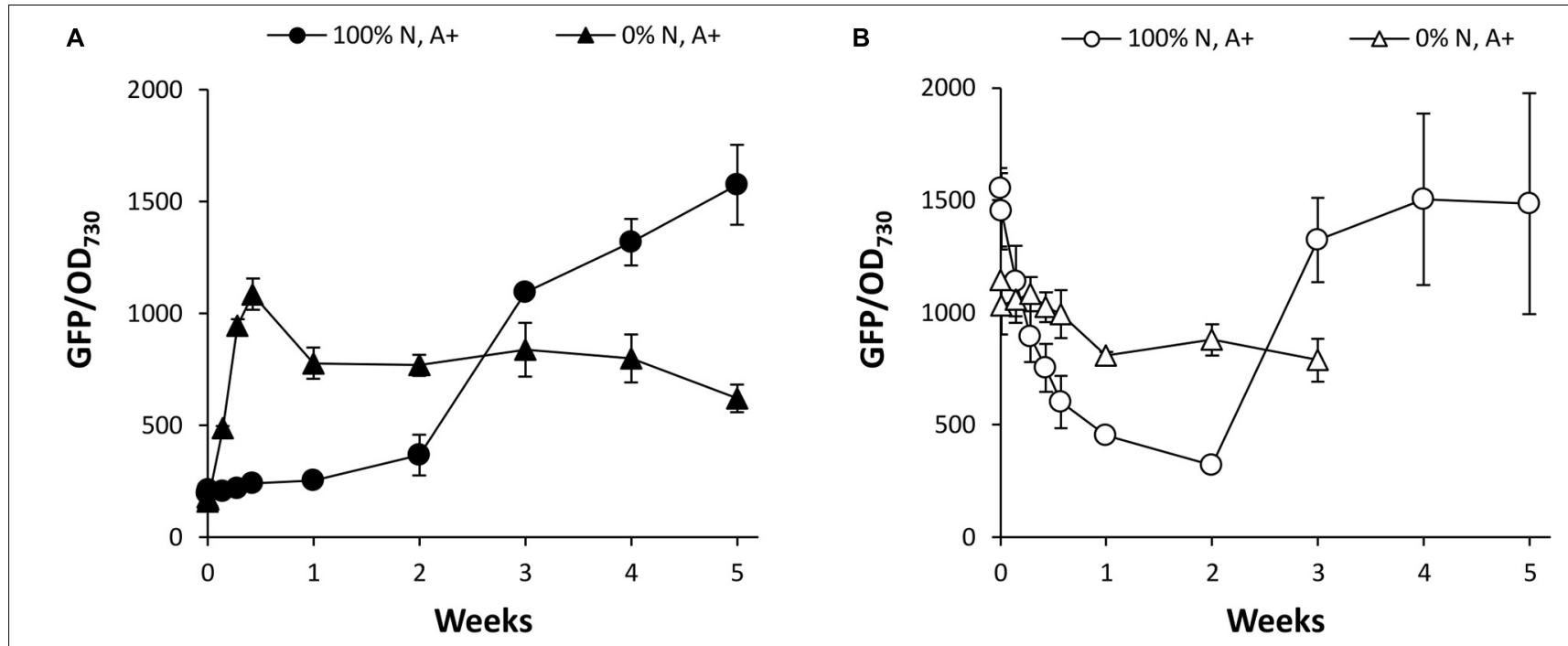

FIGURE 7 | The effect of N supply on PndbA600 activity. PndbA600 activity (GFP fluorescence normalised to OD 730 ) in Synechococcus PndbA600:GFP grown in media containing 0 or $100 \% \mathrm{~N}$ in A + background. Cultures shown in (A) were started from young, low density cultures with low promoter activity (OD < 5 , GFP $<300$, black symbols), and cultures shown in (B) were started from mature, high density cultures with high promoter activity (OD > 12, GFP > 850, white symbols). All cultures were resuspended to OD 1 in the indicated media at time point 0 . Data are means \pm S.E.M. of three independent cultures. Accompanying growth curves are presented in Supplementary Figure S6.

\section{Early Kinetics of PndbA600 Activation}

We have identified two types of media that accelerate the activation of PndbA600: the spent medium of stationary phase cultures and $\mathrm{N}$-deficient medium. The spent medium is a complex solution comprised of a combination of different levels of multiple nutrient deficiency, as well as extracellular metabolites that have been secreted by the cells throughout culture growth. To check whether $\mathrm{N}$ depletion is the cause of PndbA600 activation in the spent medium, we compared PndbA600 activation in response to the spent medium with $\mathrm{N}$-deficient medium (Figure 8). In the spent medium, PndbA600 activation is $>10$-fold faster (within $30 \mathrm{~min}$ in low to low $\mathrm{OD}$, spent media) compared to $\mathrm{N}$-deficient medium (with $24 \mathrm{~h}$ in low to low $\mathrm{OD}, 0 \% \mathrm{~N}, \mathrm{~A}+$ ). This suggests that nutrient deficiency, specifically $\mathrm{N}$ depletion, is not sufficient to explain the rapid activation in the spent medium and that other factor(s) contribute to PndbA600 activation.

\section{The Effect of Electron Transport Inhibition on PndbA600 Activity}

Type II NADH dehydrogenases play a central role in the respiratory metabolism of bacteria; however, this is not the case in cyanobacteria where NdbA function remains unclear (Howitt et al., 1999; Lea-Smith et al., 2016; Huokko et al., 2019). A role in redox sensing has been proposed (Howitt et al., 1999), so we tested the effect of changing cellular redox status on promoter activity by disturbing electron transport. For this experiment, electron transport inhibitors specific to photosynthesis [DCMU, which blocks the plastiquinone binding site of photosystem II (Duysens and Sweers, 1963)] or respiration [malonic acid (MA), which competitively inhibits succinate dehydrogenase complex II (Pardee and Potter, 1949)] were applied to low or high density

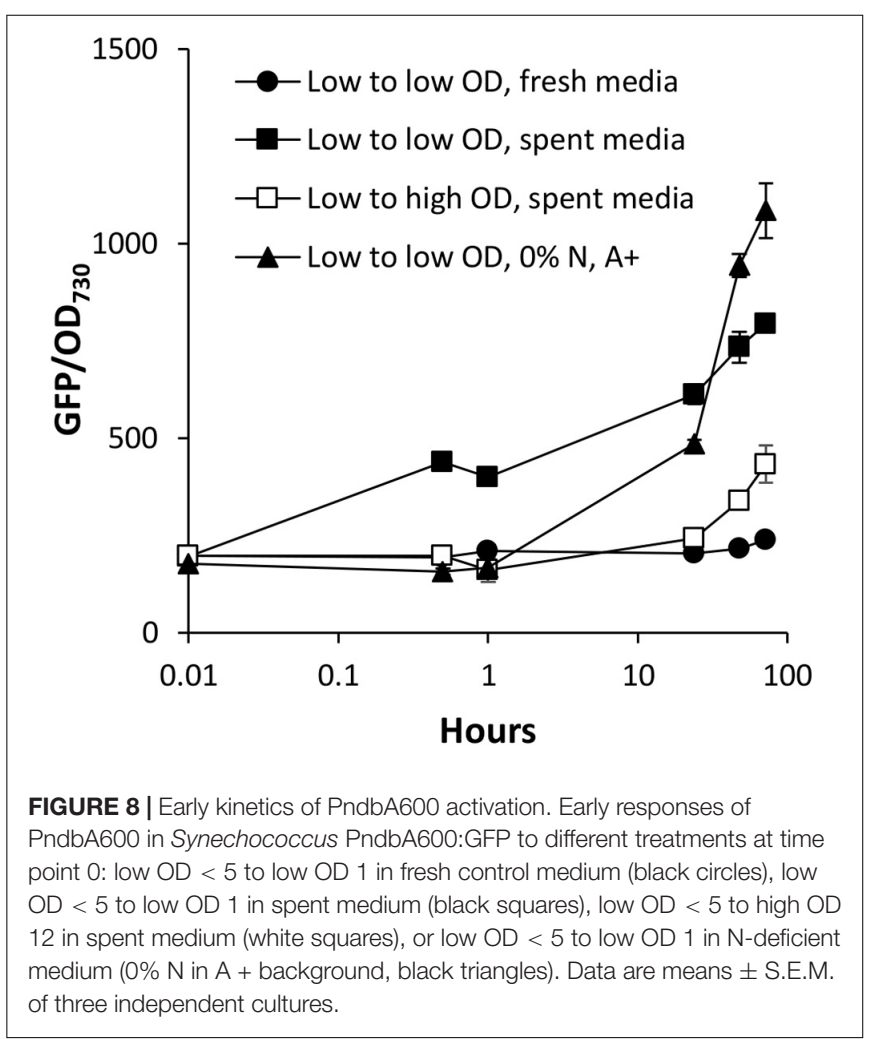

cultures of Synechococcus PndbA600:GFP grown under control conditions. Inhibitors were applied at two concentrations: a lower concentration, which allowed for growth of low density cultures $(0.1 \mu \mathrm{M}$ DCMU and $4 \mathrm{mM} \mathrm{MA})$, and a high concentration, which inhibited the growth of low density cultures $(1 \mu \mathrm{M}$ 


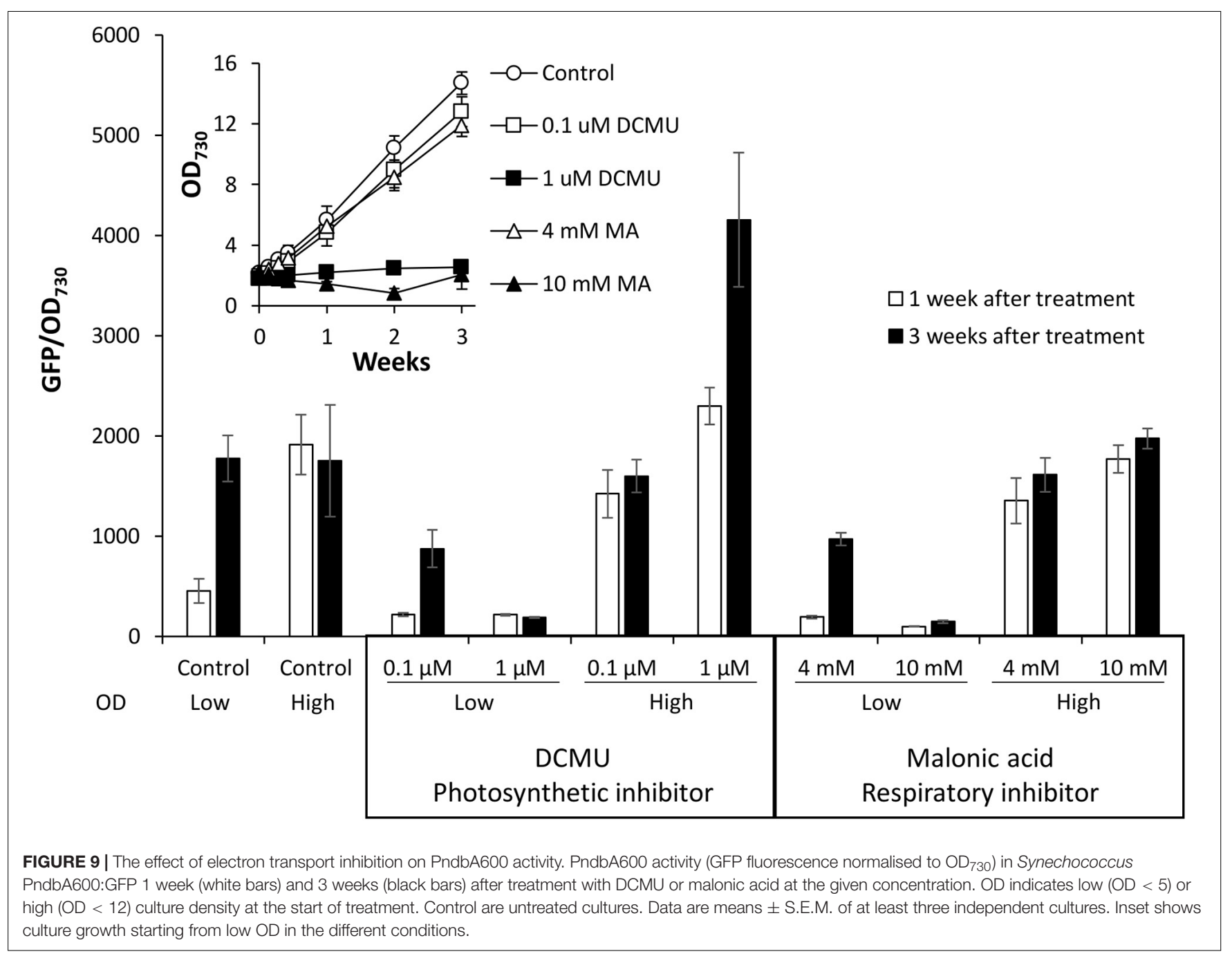

DCMU and $10 \mathrm{mM} \mathrm{MA}$; Figure 9). When the inhibitors were applied at low concentration in young, low density cultures, PndbA600 activation in the late growth phase still occurred, albeit at a lower level than without inhibitor. The slight decrease in activity may reflect the slower growth and lower density of the inhibitor-treated cultures. By contrast, high inhibitor concentrations resulted in a lack of culture growth and of PndbA600 activation. In mature, high density cultures, low concentrations of inhibitors did not alter PndbA600 activity. At high concentrations, however, DCMU increased PndbA600 activity 1.79 -fold in high density cultures whereas MA did not. These results show that the inhibition of photosynthesis, but not respiration, enhances PndbA600 activity in high density cultures.

\section{DISCUSSION}

Two-stage cultivation strategies are an attractive solution to growth/productivity trade-offs in cyanobacteria; however, the costly addition of extra steps between growth (stage I) and production (stage II) is often required to initiate stage II (Lee et al., 2012, 2016; Monshupanee et al., 2016; Kushwaha et al., 2018; Aziz et al., 2020). Less effort has been made towards utilising inherent features of cyanobacterial cultures to distinguish between stages and thus auto-induce transgene expression in stage II, cutting costs and improving economic feasibility. Auto-inducible production systems have been engineered using nutrient-deficiency responsive regulators (Liu et al., 2011; Asada et al., 2019); however, their applications may be limited by the regulatory nutrient and the timing of deficiency. Here, we instead opted to develop regulatory systems based on growth phase transitions in batch cultures and endogenous regulation by stationary phase promoters. A few studies led to the identification of cyanobacterial growth phaseresponsive genes and promoters (Foster et al., 2007; Berla and Pakrasi, 2012; Ruffing et al., 2016); however, the knowledge is still very limited for the stationary phase and its regulation in cyanobacteria. In this study, we identified a small subset of genes that specifically respond to growth phase transition in Synechocystis sp. PCC 6803. Furthermore, we report the first detailed description of the complex environmental responses of a growth phase-responsive promoter of cyanobacteria. 


\section{General Responses to Changes in Growth Phase}

Common responses of nutrient limitation studies form the main foundation of knowledge surrounding stationary phase in cyanobacteria and generally involve increased catabolism and decreased anabolism (Schwarz and Forchhammer, 2005). Direct comparisons of cyanobacterial growth phases have focussed on transcriptional responses in at most two conditions (Foster et al., 2007; Ludwig and Bryant, 2011; Berla and Pakrasi, 2012; Kopf et al., 2014). These studies have led to the identification of differentially expressed genes; however, it is difficult to differentiate between genes involved in general responses to changes in the growth phase and specific responses to the conditions in which the cultures were grown. Furthermore, it is often difficult to confirm that these datasets do in fact reflect stationary phase gene expression as low stationary phase ODs are commonly reported without any accompanying growth curves. Here, we present a comprehensive RNA sequencing dataset that enables the robust separation of growth phase-specific responses from condition-specific responses in Synechocystis sp. PCC 6803 (Supplementary Table S3). Our dataset agrees with general, growth phase-related downregulation of genes involved in photosynthesis, energy metabolism, and translation reported by previous nutrient limitation studies (Hirani et al., 2001; Richaud et al., 2001). Of the genes upregulated in the late growth phase/early stationary phase, $67 \%$ are annotated as hypothetical proteins reflecting a large gap in knowledge that persists on growth phase-specific responses in the extensively studied model cyanobacterium Synechocystis sp. PCC 6803. Besides their purpose for this study, the datasets provided in Supplementary Table S3 represent a new resource for understanding transcriptional responses of Synechocystis sp. PCC 6803 to individual nutrient deficiencies (including new transcriptomes in low $\mathrm{Mg}$ and low $\mathrm{K}$ ).

\section{Orthogonal Promoter Behaviour in Cyanobacteria}

Orthogonal molecular parts are preferred for metabolic engineering in order to avoid the interference of engineered systems by host machinery and genetic instability arising from endogenous DNA sequences (Camsund and Lindblad, 2014). Well-established prokaryotic tools such as IPTG- and tetracycline-inducible systems from E. coli perform poorly in cyanobacteria (Huang et al., 2010). Promoters derived from other species of cyanobacteria appear to have greater success despite regulatory differences between cyanobacterial species (Huang et al., 2010; Wang et al., 2012; Gordon and Pfleger, 2018). For example, the phycocyanin promoters (Pcpc) of Synechocystis sp. PCC 6803 and PCC 6714 have been used to drive heterologous expression in Synechococcus sp. PCC 7002 and PCC 7942 (Markley et al., 2015; Gao et al., 2016). Furthermore, a previous report showing higher activity of the heterologous phycocyanin promoter in the early growth phase of Synechococcus sp. PCC 7002 agrees with the expression profiles of the native phycocyanin operon in our Synechocystis sp. PCC 6803 RNAseq dataset (Madsen et al., 2018). Nevertheless, our detailed analysis of heterologous PndbA600 activity in Synechococcus sp. PCC 7002 revealed clear differences to the endogenous $n d b A$ expression profiles in Synechocystis sp. PCC 6803. While expression patterns were similar under control conditions (Figures 3B,C, 4A,B), closer examination under nutrient limitation revealed differences in correlations between the gene expression, culture density, and the growth phase in the two species (Figure 6). The promoter activation during the late growth phase observed under control conditions in this study is not an artefact of the assay, as evidenced by previous characterisation of an early growth phase-specific promoter using the same approach (Madsen et al., 2018). While this suggests that the condition-dependent activation profiles of PndbA600 should reflect promoter response to growth phase status, further studies using a truncated version of PndbA600, which has lost the ability to respond to changes in the growth phase, will strengthen this finding. This study, therefore, highlights the importance of thorough characterisation of molecular components to enable rational design and accurate prediction of the behaviour of more complex assemblies in non-standard conditions.

\section{Environmental Regulation of PndbA600}

This study also presents the first detailed analysis of a growth phase-responsive promoter of cyanobacteria. Responses to changing environmental stimuli, including culture density, growth media, nutrient availability, and cellular redox status, showed differing requirements for PndbA600 activation and deactivation (Figure 10). PndbA600 activation could be induced either by lowering $\mathrm{N}$ supply or presenting Factor $\mathrm{X}$ from the spent medium of stationary phase cultures. Interestingly, culture density per se had no effect on PndbA600 activation, but low culture density was required for PndbA600 deactivation in addition to sufficient $\mathrm{N}$ supply and the absence of Factor $\mathrm{X}$. Furthermore, PndbA600 may respond in a dose-dependent manner to Factor X. Increasing the relative amount of Factor X per cell may have a proportionate effect on PndbA600 activity. For example, PndbA600 activation in spent media was $>100$-fold faster in young cultures resuspended at low density compared to high density (Figures 5B, 8). PndbA600 activity in spent media was also higher in mature cultures resuspended at low density compared to high density (Figure 5D). Similarly, mature cultures transferred from spent to $\mathrm{N}$-deficient media showed a visible reduction in GFP fluorescence, which could be due to the removal of Factor X (Figure 7B). Additional analyses are required to identify Factor $\mathrm{X}$, which could be either a downstream response to $\mathrm{N}$ limitation or an unrelated extracellular metabolite e.g., signalling molecule secreted by stationary phase cells. Compositional analyses of spent media fractions paired with gene expression and promoter analyses could yield further insights into stationary phase and its regulation in cyanobacteria.

While heterologous promoter activity may not accurately reflect endogenous transcriptional regulation, this study could provide initial insights into growth phase-specific regulation and NdbA function in cyanobacteria. The $n d b A$ gene encodes a type II NADH dehydrogenase of unknown function. The ability of $n d b A$ knockout strains of Synechocystis sp. PCC 6803 to grow under otherwise lethal high-light conditions in 


\section{Activation}

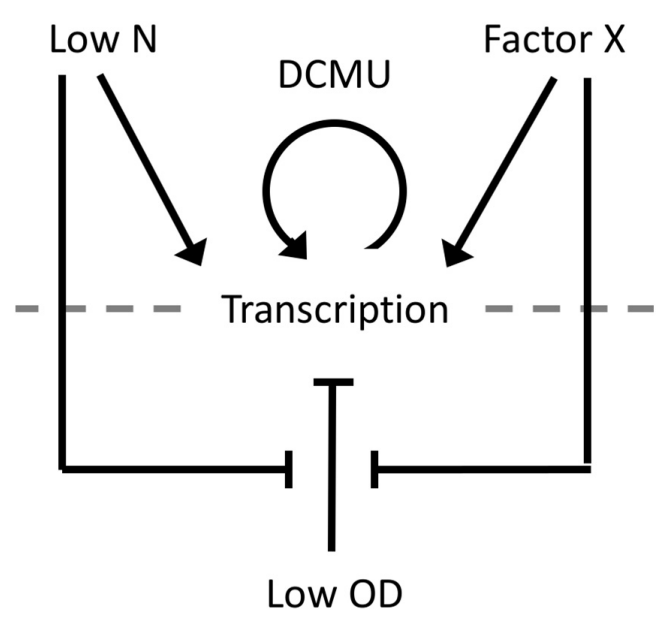

\section{Deactivation}

FIGURE 10 | Model of the environmental regulation of PndbA600. Promoter activation and, thus, transcription occur in response to either low $\mathrm{N}$ or Factor $X$ of the spent stationary phase medium. Once activated, promoter activity can be further increased using the photosynthesis-specific inhibitor DCMU. Promoter deactivation requires low culture density, sufficient $\mathrm{N}$ supply, and the absence of Factor $X$.

a PSI-less background led to the suggestion of a regulatory role for $\mathrm{NdbA}$ and monitoring of, e.g., cellular redox status (Howitt et al., 1999). In support of these functions, native NdbA localises to the thylakoid membrane (Huokko et al., 2019), and the PndbA600 promoter responds to disturbances in electron transport in the heterologous expression host (Figure 9). Specifically, heterologous PndbA600 responds to photosynthetic, but not respiratory, inhibition, whereby the key difference is the reduction of NADP + in photosynthesis and oxidation of $\mathrm{NADH}$ in respiration. These findings, paired with the presence of $\mathrm{NADH}$ binding motifs in the $n d b A$ coding sequence (Howitt et al., 1999), suggest that $\mathrm{NAD}(\mathrm{P})+/ \mathrm{NAD}(\mathrm{P}) \mathrm{H}$ balance may be an important factor regulating PndbA600/NdbA activity levels.

\section{Industrial Applications}

This study describes first steps towards developing regulatory systems to drive stage II of a two-stage cultivation system in cyanobacteria. Libraries of stage II promoters can now be constructed based on PndbA600 or other late growth phase-responsive promoters and, subsequently, used to optimise heterologous metabolic pathways for industrial production or fine-tune endogenous metabolic pathways supplying precursors necessary for the engineered process. Similarly, libraries of stage I promoters can be constructed to optimise growth, potentially improving growth rates and thus decreasing time scales until the initiation of stage II. Furthermore, many additional analyses can be performed on the RNAseq dataset to identify genes/promoters with any combination of growth phase- and/or nutrientspecific activity for diverse applications in industry, e.g., biosensors.

Stationary phase may not be suitable for all industrial applications or commercial products, and therefore, a careful selection of products and processes is important. While general decreases in anabolism occurs during the stationary phase, select processes continue at appreciable levels even after prolonged starvation (Schwarz and Forchhammer, 2005). Notable examples are secondary metabolites important for human health, particularly as anti-infective drugs such as antibiotics (Ruiz et al., 2010). Here, we used a new approach for the robust separation of growth phase- vs. condition-specific processes. This approach can also complement bioprospecting for new secondary metabolites in cyanobacteria and other microorganisms by comparing transcriptomic and metabolomic data across a variety of conditions to identify genes and unravel biosynthetic pathways underpinning the production of interesting metabolites. The large proportion (67\%) of late growth phase-specific genes encoding hypothetical proteins identified in Synechocystis sp. PCC 6803 highlights the strength of this approach, as well as the great potential for the identification of new cyanobacterial products and pathways.

As a chassis, Synechococcus sp. PCC 7002 has numerous advantages for industrial production, including relatively fast growth rates and high tolerance to various parameters such as light, temperature, and salinity (Nomura et al., 2006). Another desirable feature we have observed in this strain is sedimentation in the stationary phase, which allows for easy biomass harvest at the end of stage II without the need for energy-demanding techniques such as centrifugation (data not shown). Perhaps, the most sustainable application of PndbA600driven two-stage cultivation strategies involves seeding the engineered Synechococcus cultures in the fresh growth medium, biomass accumulation during stage I until nutrient depletion results in the auto-induction of stage II, and finally applicationspecific downstream processing of the biomass and supernatant. Recycling stage II cells to seed new cultures is not ideal as we observed a decrease in the amount of biomass attained, no increase in promoter activity, and no decrease in time to stage II (high to low OD in fresh media, Supplementary Figure S5C). By contrast, maximum biomass can be further increased by concentrating low density cultures in fresh nutrients to generate higher culture densities compared to control conditions (low to high OD in fresh media, Supplementary Figure S5A). Alternatively, if the expense of time is greater than the benefit of high biomass, stage II can be induced at lower culture densities by using nutrient limitation to significantly reduce timescales. Early induction could prove particularly profitable if nutrient-specific responses increase the productivity of stage II cells. Finally, stage II productivity can be further improved by increasing the activity of the auto-inducible promoter, either by engineering PndbA600 or adding supplements such as DCMU.

This study provides the first insights into the regulation of stationary phase in cyanobacteria. Additional studies to identify DNA motifs present within growth phase-responsive 
promoters, transcription factors that bind to these motifs, and other regulatory molecules will provide further important insights to this still elusive phase of cyanobacteria. Unravelling these mysteries and expanding the foundation of knowledge surrounding these organisms will be of great value to both academia and industry.

\section{DATA AVAILABILITY STATEMENT}

The datasets presented in this study can be found in online repositories. The names of the repository/repositories and accession number(s) can be found below: https://www.ebi.ac.uk/ ena, PRJEB40560.

\section{AUTHOR CONTRIBUTIONS}

MAM and AA designed the study and wrote the manuscript. MAM performed the experiments. MAM, GH, $\mathrm{PH}$, and AA analysed the data. All authors contributed to the article and approved the submitted version.

\section{REFERENCES}

Abisado, R. G., Benomar, S., Klaus, J. R., Dandekar, A. A., and Chandler, J. R. (2018). Bacterial quorum sensing and microbial community interactions. mBio 9:e02331-17. doi: 10.1128/mBio.02331-17

Al-Haj, L., Lui, Y. T., Abed, R. M., Gomaa, M. A., and Purton, S. (2016). Cyanobacteria as chassis for industrial biotechnology: progress and prospects. Life 6:42. doi: 10.3390/life6040042

Amezaga, J. M., Amtmann, A., Biggs, C. A., Bond, T., Gandy, C. J., Honsbein, A., et al. (2014). Biodesalination: a case study for applications of photosynthetic bacteria in water treatment. Plant Physiol. 164, 1661-1676. doi: 10.1104/pp.113. 233973

Andersen, C. L., Jensen, J. L., and Ørntoft, T. F. (2004). Normalization of real-time quantitative reverse transcription-PCR data: a model-based variance estimation approach to identify genes suited for normalization, applied to bladder and colon cancer data sets. Cancer Res. 64, 5245-5250. doi: 10.1158/0008-5472.can04-0496

Asada, R., Shiraiwa, Y., and Suzuki, I. (2019). A novel cell lysis system induced by phosphate deficiency in the Cyanobacterium Synechocystis sp. PCC 6803. J. Appl. Phycol. 31, 1069-1076. doi: 10.1007/s10811-018-1652-6

Aziz, M. M. A., Kassim, K. A., Shokravi, Z., Jakarni, F. M., Liu, H. Y., Zaini, N., et al. (2020). Two-stage cultivation strategy for simultaneous increases in growth rate and lipid content of microalgae: a review. Renew. Sustain. Energy Rev. 119:109621. doi: 10.1016/j.rser.2019.109621

Berla, B. M., and Pakrasi, H. B. (2012). Upregulation of plasmid genes during stationary phase in Synechocystis sp. strain PCC 6803, a Cyanobacterium. Appl. Environ. Microbiol. 78, 5448-5451. doi: 10.1128/aem.01174-1112

Berla, B. M., Saha, R., Immethun, C. M., Maranas, C. D., Moon, T. S., and Pakrasi, H. B. (2013). Synthetic biology of Cyanobacteria: unique challenges and opportunities. Front. Microbiol. 4:246. doi: 10.3389/fmicb.2013.00246

Burg, J. M., Cooper, C. B., Ye, Z., Reed, B. R., Moreb, E. A., and Lynch, M. D. (2016). Large-scale bioprocess competitiveness: the potential of dynamic metabolic control in two-stage fermentations. Curr. Opin. Chem. Eng. 14, 121-136. doi: 10.1016/j.coche.2016.09.008

Camsund, D., and Lindblad, P. (2014). Engineered transcriptional systems for Cyanobacterial biotechnology. Front. Bioeng. Biotechnol. 2:40. doi: 10.3389/ fbioe.2014.00040

Clark, R. L., McGinley, L. L., Purdy, H. M., Korosh, T. C., Reed, J. L., Root, T. W., et al. (2018). Light-optimized growth of Cyanobacterial cultures: growth

\section{FUNDING}

This work was supported by the Engineering and Physical Sciences Research Council (EPSRC; EP/J004871/1) and Biotechnology and Biological Sciences Research Council (BBSRC; IBCarb-BIV-0316-015 and BB/R019894/1). The Glasgow Polyomics facility was supported by the Wellcome Trust (105614/Z/14/Z).

\section{ACKNOWLEDGMENTS}

We are grateful to Linda Lawton (Robert Gordon University, United Kingdom) for providing the Synechocystis sp. PCC 6803 strain.

\section{SUPPLEMENTARY MATERIAL}

The Supplementary Material for this article can be found online at: https://www.frontiersin.org/articles/10.3389/fbioe. 2020.619055/full\#supplementary-material

phases and productivity of biomass and secreted molecules in light-limited batch growth. Metab. Eng. 47, 230-242. doi: 10.1016/j.ymben.2018.03.017

Dittmann, E., Gugger, M., Sivonen, K., and Fewer, D. P. (2015). Natural product biosynthetic diversity and comparative genomics of the Cyanobacteria. Trends Microbiol. 23, 642-652. doi: 10.1016/j.tim.2015.07.008

Duysens, L. N. M., and Sweers, H. E. (1963). "Mechanism of the two photochemical reactions in alage as studied by means of fluorescence," in Studies on Microalgae and Photosynthetic Bacteria, ed. S. Miyachi (Tokyo: University of Tokyo Press), 353-372.

Esteves-Ferreira, A. A., Inaba, M., Obata, T., Fort, A., Fleming, G. T. A., Araújo, W. L., et al. (2017). A Novel mechanism, linked to cell density, largely controls cell division in Synechocystis. Plant Physiol. 174, 2166-2182. doi: 10.1104/pp.17. 00729

Foster, J. S., Singh, A. K., Rothschild, L. J., and Sherman, L. A. (2007). Growthphase dependent differential gene expression in Synechocystis sp. strain PCC 6803 and regulation by a group 2 sigma factor. Arch. Microbiol. 187, 265-279. doi: 10.1007/s00203-006-0193-196

Gale, G. A. R., Schiavon Osorio, A. A., Mills, L. A., Wang, B., Lea-Smith, D. J., and McCormick, A. J. (2019). Emerging species and genome editing tools: future prospects in Cyanobacterial synthetic biology. Microorganisms 7:409. doi: 10.3390/microorganisms7100409

Gao, X., Gao, F., Liu, D., Zhang, H., Nie, X., and Yang, C. (2016). Engineering the methylerythritol phosphate pathway in Cyanobacteria for photosynthetic isoprene production from $\mathrm{CO}_{2}$. Energy Environ. Sci. 9, 1400-1411. doi: 10.1039/ C5EE03102H

Gordon, G. C., and Pfleger, B. F. (2018). Regulatory tools for controlling gene expression in Cyanobacteria. Adv. Exp. Med. Biol. 1080, 281-315. doi: 10.1007/ 978-981-13-0854-3_12

Hirani, T. A., Suzuki, I., Murata, N., Hayashi, H., and Eaton-Rye, J. J. (2001). Characterization of a two-component signal transduction system involved in the induction of alkaline phosphatase under phosphate-limiting conditions in Synechocystis sp. PCC 6803. Plant Mol Biol. 45, 133-144. doi: 10.1023/a: 1006425214168

Howitt, C. A., Udall, P. K., and Vermaas, W. F. (1999). Type 2 NADH dehydrogenases in the Cyanobacterium Synechocystis sp. strain PCC 6803 are involved in regulation rather than respiration. J. Bacteriol. 181, 3994-4003. doi: 10.1128/jb.181.13.3994-4003.1999

Hu, J., and Wang, Q. (2018). Regulatory sRNAs in Cyanobacteria. Front. Microbiol. 9:2399. doi: 10.3389/fmicb.2018.02399 
Huang, H. H., Camsund, D., Lindblad, P., and Heidorn, T. (2010). Design and characterization of molecular tools for a synthetic biology approach towards developing Cyanobacterial biotechnology. Nucleic Acids Res. 38, 2577-2593. doi: 10.1093/nar/gkq164

Huokko, T., Muth-Pawlak, D., and Aro, E. M. (2019). Thylakoid localized Type $2 \mathrm{NAD}(\mathrm{P}) \mathrm{H}$ dehydrogenase $\mathrm{NdbA}$ optimizes light-activated heterotrophic growth of Synechocystis sp. PCC 6803. Plant Cell Physiol. 60, 1386-1399. doi: 10.1093/pcp/pcz044

Knoop, H., and Steuer, R. (2015). A computational analysis of stoichiometric constraints and trade-offs in Cyanobacterial biofuel production. Front. Bioeng. Biotechnol. 3:47. doi: 10.3389/fbioe.2015.00047

Kopf, M., Klähn, S., Scholz, I., Matthiessen, J. K., Hess, W. R., and Voß, B. (2014). Comparative analysis of the primary transcriptome of Synechocystis sp. PCC 6803. DNA Res. 21, 527-539. doi: 10.1093/dnares/dsu018

Kushwaha, D., Upadhyay, S. N., and Mishra, P. K. (2018). Growth of Cyanobacteria: optimization for Increased carbohydrate content. Appl. Biochem. Biotechnol. 184, 1247-1262. doi: 10.1007/s12010-017-2620-2623

Lea-Smith, D. J., Bombelli, P., Vasudevan, R., and Howe, C. J. (2016). Photosynthetic, respiratory and extracellular electron transport pathways in Cyanobacteria. Biochim. Biophys. Acta Bioenerget. 1857, 247-255. doi: 10.1016/ j.bbabio.2015.10.007

Lee, M. C., Chen, Y. C., and Peng, T. C. (2012). Two-stage culture method for optimized polysaccharide production in Spirulina platensis. J. Sci. Food Agric. 92, 1562-1569. doi: 10.1002/jsfa.4743

Lee, S. H., Lee, J. E., Kim, Y., and Lee, S. Y. (2016). The production of high purity Phycocyanin by Spirulina platensis using light-emitting diodes based two-stage cultivation. Appl. Biochem. Biotechnol. 178, 382-395. doi: 10.1007/s12010-0151879- 1875

Liu, X., Fallon, S., Sheng, J., and Curtiss, R. III (2011). $\mathrm{CO}_{2}$-limitation-inducible green recovery of fatty acids from Cyanobacterial biomass. Proc. Natl. Acad. Sci. U.S.A. 108, 6905-6908. doi: 10.1073/pnas. 1103016108

Ludwig, M., and Bryant, D. A. (2011). Transcription profiling of the model Cyanobacterium Synechococcus sp. Strain PCC 7002 by Next-Gen (SOLiD ${ }^{\mathrm{TM}}$ ) sequencing of cDNA. Front. Microbiol. 2:41. doi: 10.3389/fmicb.2011.00041

Madsen, M. A., Semerdzhiev, S., Amtmann, A., and Tonon, T. (2018). Engineering mannitol biosynthesis in Escherichia coli and Synechococcus sp. PCC 7002 using a green algal fusion protein. ACS Synth. Biol. 7, 2833-2840. doi: 10.1021/ acssynbio. 8 b00238

Markley, A. L., Begemann, M. B., Clarke, R. E., Gordon, G. C., and Pfleger, B. F. (2015). Synthetic biology toolbox for controlling gene expression in the Cyanobacterium Synechococcus sp. strain PCC 7002. ACS Synth. Biol. 4, 595-603. doi: 10.1021/sb500260k

Miao, R., Xie, H., Liu, X., Lindberg, P., and Lindblad, P. (2020). Current processes and future challenges of photoautotrophic production of acetyl-CoA-derived solar fuels and chemicals in Cyanobacteria. Curr. Opin. Chem. Biol. 59, 69-76. doi: 10.1016/j.cbpa.2020.04.013

Miksch, G., Bettenworth, F., Friehs, K., Flaschel, E., Saalbach, A., Twellmann, T., et al. (2005). Libraries of synthetic stationary-phase and stress promoters as a tool for fine-tuning of expression of recombinant proteins in Escherichia coli. J. Biotechnol. 120, 25-37. doi: 10.1016/j.jbiotec.2005.04.027

Monshupanee, T., Nimdach, P., and Incharoensakdi, A. (2016). Two-stage (photoautotrophy and heterotrophy) cultivation enables efficient production of bioplastic poly-3-hydroxybutyrate in auto-sedimenting Cyanobacterium. Sci. Rep. 6:37121. doi: 10.1038/srep37121

Nomura, C. T., Sakamoto, T., and Bryant, D. A. (2006). Roles for hemecopper oxidases in extreme high-light and oxidative stress response in the Cyanobacterium Synechococcus sp. PCC 7002. Arch. Microbiol. 185, 471-479. doi: 10.1007/s00203-006-0107-107

Nyström, T. (2004). Stationary-phase physiology. Annu. Rev. Microbiol. 58, 161181. doi: 10.1146 /annurev.micro.58.030603.123818

Pardee, A. B., and Potter, V. R. (1949). Malonate inhibition of oxidations in the Krebs tricarboxylic acid cycle. J. Biol. Chem. 178, 241-250.

Pierobon, S. C., Cheng, X., Graham, P. J., Nguyen, B., Karakolis, E. G., and Sinton, D. (2018). Emerging microalgae technology: a review. Sustain. Energy Fuels 2, 13-38. doi: 10.1039/C7SE00236J

Pinto, F., Pacheco, C. C., Ferreira, D., Moradas-Ferreira, P., and Tamagnini, P. (2012). Selection of suitable reference genes for RT-qPCR analyses in Cyanobacteria. PLoS One 7:e34983. doi: 10.1371/journal.pone.0034983
Richaud, C., Zabulon, G., Joder, A., and Thomas, J. C. (2001). Nitrogen or sulfur starvation differentially affects phycobilisome degradation and expression of the nblA gene in Synechocystis strain PCC 6803. J. Bacteriol. 183, 2989-2994. doi: $10.1128 / \mathrm{jb}$.183.10.2989-2994.2001

Ruffing, A. M., Jensen, T. J., and Strickland, L. M. (2016). Genetic tools for advancement of Synechococcus sp. PCC 7002 as a Cyanobacterial chassis. Microb. Cell Fact 15:190. doi: 10.1186/s12934-016-0584-586

Ruiz, B., Chávez, A., Forero, A., García-Huante, Y., Romero, A., Sánchez, M., et al. (2010). Production of microbial secondary metabolites: regulation by the carbon source. Crit. Rev. Microbiol. 36, 146-167. doi: 10.3109/ 10408410903489576

Santos-Merino, M., Singh, A. K., and Ducat, D. C. (2019). New applications of synthetic biology tools for Cyanobacterial metabolic engineering. Front. Bioeng. Biotechnol. 7:33. doi: 10.3389/fbioe.2019.00033

Schuurmans, R. M., Matthijs, J. C., and Hellingwerf, K. J. (2017). Transition from exponential to linear photoautotrophic growth changes the physiology of Synechocystis sp. PCC 6803. Photosynth. Res. 132, 69-82. doi: 10.1007/s11120016-0329-328

Schwarz, R., and Forchhammer, K. (2005). Acclimation of unicellular Cyanobacteria to macronutrient deficiency: emergence of a complex network of cellular responses. Microbiology 151(Pt 8), 2503-2514. doi: 10.1099/mic.0.27883-27880

Singh, J. S., Kumar, A., Rai, A. N., and Singh, D. P. (2016). Cyanobacteria: a precious bio-resource in agriculture, ecosystem, and environmental sustainability. Front. Microbiol. 7:529. doi: 10.3389/fmicb.2016. 00529

Singh, R., Parihar, P., Singh, M., Bajguz, A., Kumar, J., Singh, S., et al. (2017). Uncovering potential applications of Cyanobacteria and algal metabolites in biology, agriculture and medicine: current status and future prospects. Front. Microbiol. 8:515. doi: 10.3389/fmicb.2017.00515

Stanier, R. Y., Kunisawa, R., Mandel, M., and Cohen-Bazire, G. (1971). Purification and properties of unicellular blue-green algae (order Chroococcales). Bacteriol. Rev. 35, 171-205. doi: 10.1128/mmbr.35.2.171-205.1971

Stevens, S. E., and Porter, R. D. (1980). Transformation in Agmenellum quadruplicatum. Proc. Natl. Acad. Sci. U.S.A. 77, 6052-6056. doi: 10.1073/pnas. 77.10 .6052

Testa, R. L., Delpino, C., Estrada, V., and Diaz, M. S. (2019). “"Bioethanol production with Cyanobacteria by a two-stage fermentation strategy," in Computer Aided Chemical Engineering, eds A. A. Kiss, E. Zondervan, R. Lakerveld, and L. Özkan (Amsterdam: Elsevier), 499-504. doi: 10.1016/b9780-12-818634-3.50084-9

Thajuddin, N., and Subramanian, G. (2005). Cyanobacterial biodiversity and potential applications in biotechnology. Curr. Sci. 89, 47-57.

Trapnell, C., Roberts, A., Goff, L., Pertea, G., Kim, D., Kelley, D. R., et al. (2012). Differential gene and transcript expression analysis of RNA-seq experiments with TopHat and cufflinks. Nat. Protoc. 7, 562-578. doi: 10.1038/nprot.2012. 016

Untergasser, A., Cutcutache, I., Koressaar, T., Ye, J., Faircloth, B. C., Remm, M., et al. (2012). Primer3-new capabilities and interfaces. Nucleic Acids Res. 40:e115. doi: 10.1093/nar/gks596

Wang, B., Wang, J., Zhang, W., and Meldrum, D. R. (2012). Application of synthetic biology in Cyanobacteria and algae. Front. Microbiol. 3:344. doi: 10. 3389/fmicb.2012.00344

Xiong, W., Cano, M., Wang, B., Douchi, D., and Yu, J. (2017). The plasticity of Cyanobacterial carbon metabolism. Curr. Opin. Chem. Biol. 41, 12-19. doi: 10.1016/j.cbpa.2017.09.004

Conflict of Interest: The authors declare that the research was conducted in the absence of any commercial or financial relationships that could be construed as a potential conflict of interest.

Copyright (c) 2021 Madsen, Hamilton, Herzyk and Amtmann. This is an openaccess article distributed under the terms of the Creative Commons Attribution License (CC BY). The use, distribution or reproduction in other forums is permitted, provided the original author(s) and the copyright owner(s) are credited and that the original publication in this journal is cited, in accordance with accepted academic practice. No use, distribution or reproduction is permitted which does not comply with these terms. 